\title{
Strength of Thin-Walled Lipped Channel Section Columns with Shell-Shaped Curved Grooves
}

\author{
Koki Hoshide, Mitao Ohga', Pang-jo Chun'1, Tsunemi Shigematsu², Sinichi Kawamura ${ }^{3}$ \\ ${ }^{1}$ Department of Civil \& Environmental Engineering, Ehime University, Matsuyama, Japan \\ ${ }^{2}$ Department of Technology, Tokuyama College, Tokuyama, Japan \\ ${ }^{3}$ Department of Kure, College of Technology, Kure, Japan \\ Email: b861001u@mails.cc.ehime-u.ac.jp
}

How to cite this paper: Hoshide, K., Ohga, M., Chun, P.-j., Shigematsu, T. and Kawamura, S. (2018) Strength of Thin-Walled Lipped Channel Section Columns with Shell-Shaped Curved Groov. Open Journal of Civil Engineering, 8, 508-523. https://doi.org/10.4236/ojce.2018.84036

Received: May 19, 2018

Accepted: December 10, 2018

Published: December 13, 2018

Copyright (C) 2018 by authors and Scientific Research Publishing Inc. This work is licensed under the Creative Commons Attribution International License (CC BY 4.0).

http://creativecommons.org/licenses/by/4.0/

\section{Open Access}

\begin{abstract}
Thin-walled member is structurally superior to a construction member. However, by reason of complexity in structure the stress and the deformation to yield the cross section are complicated. Specially, in case thin-walled members, such as thin-walled channel section columns, which are subjected to compressive force, these members produce the local buckling, distortional buckling and overall buckling. A number of experimental and theoretical investigations subjected to axial compressive force are generated for thin-walled channel section columns with triangle-shaped folded groove by Hancock [1] and with complex edge stiffeners and web stiffeners by Wang [2]. In case thin-walled channel section column with folded groove which is subjected to axial compressive force, it is cleared that the buckling mode shapes are ordinarily generated for local buckling mode shape of plate-panel composing cross section of member in short member aspect ratio and overall buckling mode shape as column and distortional buckling mode shape interacting between local buckling and overall buckling similarly normal thin-walled member. It is cleared analytically and experimentally that buckling strength and critical strength of thin-walled channel section column with folded groove can increase sharply in comparison with that of normal thin-walled member composing only plate-panel. In this paper a new cross section of shell-shaped curved groove [3] was proposed instead of the thin-walled lipped channel section column with triangle- and rectangle-shaped folded grooves used ordinarily, and therefore the comparison and the examination of buckling strength and buckling behavior were generated in the case of preparing triangle-shaped folded and shell-shaped curved grooves to web and flange of thin-walled channel section column. And then in order to investigate the buckling behavior on the thin-walled channel section column with folded and curved grooves, exact buckling strength and the buckling mode
\end{abstract}


shapes are generated by using the transfer matrix method. The analytical local distortional and overall elastic buckling loads of thin-walled channel section column with folded and curved grooves can be obtained simultaneously by use of the transfer matrix method. Furthermore, a technique to estimate the buckling mode shapes of these members is also shown.

\section{Keywords}

Transfer Matrix Method, Folded and Curved Grooves, Local, Distortional and Overall Buckling Strength, Buckling Mode Shapes

\section{Introduction}

Thin-walled member is structurally superior to a construction member. However by reason of complexity in structure the stress and the deformation to yield the cross section are complicated.

Specially, in case thin-walled members, such as thin-walled channel section columns, which are subjected to compressive force, the members with folded grooves produce the local buckling, distortional buckling and overall buckling.

In recent year, thin-walled channel section columns with folded grooves fabricated from cold-reduced high strength steel plate have been used by advance of manufacturing technique. However, a design of thin-walled member with folded grooves subjected to axial force is generated by obtained strength during assumed bending test as use of deck-plate. And experimental and theoretical investigations are extremely insufficient for various complicated buckling behavior above-mentioned. Especially, in the case of thin-walled member with folded and curved grooves subjected to axial force, the examination of most effective shape to cross section is present state to be not almost investigated.

A number of experimental and theoretical investigations subjected to axial compressive force are performed for thin-walled channel section column with triangle-shaped folded groove by Hancock [1] and Wang [2]. In case thin-walled channel section column with folded groove which is subjected to axial compressive force, it is cleared that the buckling mode shapes are ordinarily generated for local buckling mode shape of plate-panel composing cross section of member in short member aspect ratio and overall buckling mode shape as column and distortional buckling mode shape interacting between local buckling and overall buckling like similarly normal thin-walled member.

It is cleared analytically and experimentally that the buckling strength and the critical strength of thin-walled channel section column with folded groove can increase sharply in comparison with normal thin-walled member composing only plate-panels.

Further, on the position, the number and the size of groove-cross section and the effect exerting buckling behavior and buckling strength are examined. Then, 
it is clear that more large stiffened effect is obtained by establishing groove-cross section not only web but also flanges.

As regards the investigation of the shape of folded groove, the comparison and the examination on the effect exerting bending strength of deck-plate during the shape of folded groove are reported in the case of using deck-plate the thin-walled channel section column with triangle- and rectangle-shaped grooves.

This study aims to propose a new cross section of shell-shaped curved groove [3] instead of the thin-walled channel section column with a triangle-shaped folded groove used ordinarily, and therefore the comparison and the examination of buckling strength and buckling behavior are generated in the case of preparing triangle-shaped folded and shell-shaped curved grooves to web and flange of thin-walled channel section column.

And then in order to investigate the buckling behavior on the thin-walled channel section column with folded and curved grooves, exact buckling strength and the buckling mode shapes are generated by using the transfer matrix method. In analysis the transfer equations are proposed by considering the compatibility and the equilibrium conditions between plate-panel and groove. The analytical local, distortional and overall elastic buckling loads of thin-walled channel section column with folded and curved grooves can be obtained simultaneously by use of the transfer matrix method. Furthermore, a technique to estimate the buckling mode shapes of these members is also shown.

\section{Analytical Theory [4] [5]}

\subsection{Field Transfer Matrix for Shell-Panel}

From the equilibrium equations of forces for the shell-panel subjected to in-plane compressive force (Figure 1) and from the relation between strains and deformations for shell-panel, the partial differential equations for the state variables ${ }^{*} w_{S},{ }^{*} \varphi_{S \varphi},{ }^{*} M_{S \varphi},{ }^{*} V_{S \varphi},{ }^{*} v_{S},{ }^{*} u_{S},{ }^{*} N_{S \varphi}$ and ${ }^{*} N_{S \varphi x}$ are obtained. By substituting the extended state variables on the basis of condition as the simple support
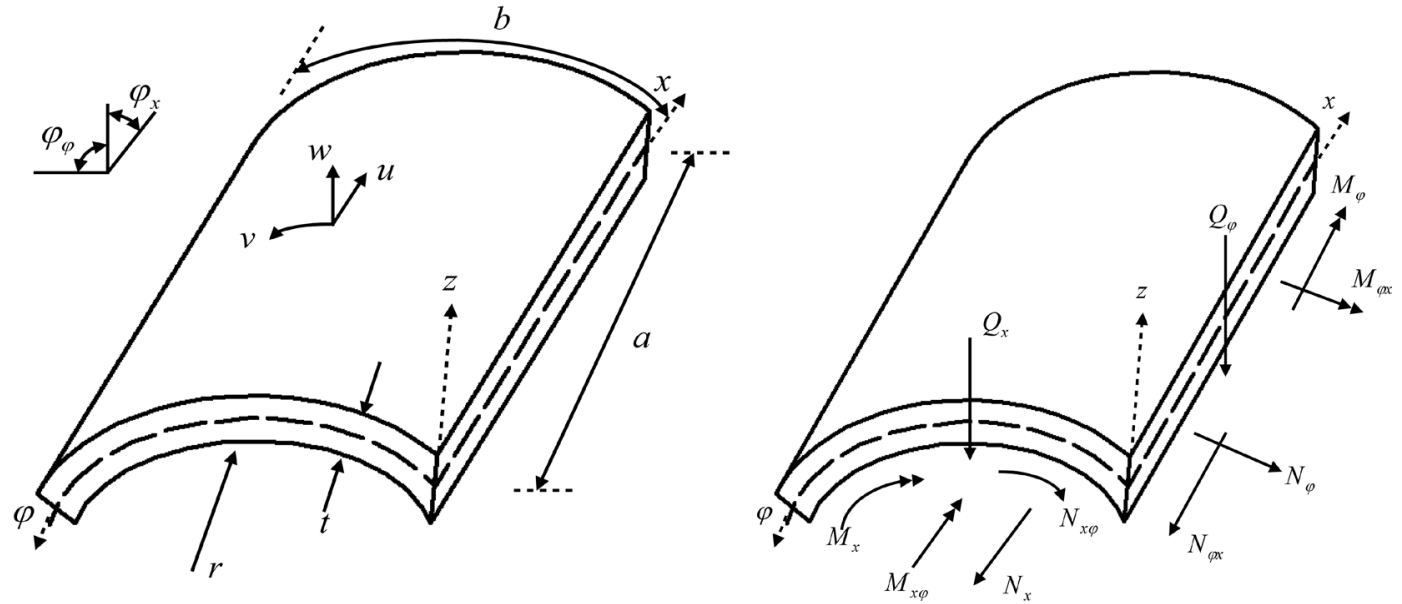

Figure 1. Forces and deformation of shell-panel subjected to in-plane compressive load. 
by the sides $x=0$ and $x=L$ of the shell-pate into the partial differential equations, the following ordinary differential equations

$$
\frac{\mathrm{d} Z_{S}}{r \mathrm{~d} \varphi}=Z_{S}^{\cdot}=\left[{ }^{*}{w_{S}}_{S},{ }^{*} \varphi_{S \varphi}^{\cdot},{ }^{*} M_{S \varphi},{ }^{*} V_{S \varphi}^{\cdot},{ }^{*} v_{S}^{\cdot},{ }^{*} u_{S}^{\cdot},{ }^{*} N_{S \varphi}^{\cdot},{ }^{*} N_{S \varphi x}\right]
$$

referenced to the variable $\varphi$ are only obtained.

$$
\left(\begin{array}{c}
{ }^{*} w_{S} \\
{ }^{*} \varphi_{S \varphi} \\
{ }^{*} M_{S \varphi} \\
{ }^{*} V_{S \varphi} \\
{ }^{*} v_{S} \\
{ }^{*} u_{S} \\
{ }^{*} N_{S \varphi} N_{S \varphi x}
\end{array}\right)=\left(\begin{array}{cccccccc}
0 & -\alpha & 0 & 0 & \frac{1}{r} & 0 & 0 & 0 \\
-\frac{\alpha K_{21}}{K_{22}} & 0 & \frac{\alpha K_{0}}{K_{22}} & 0 & 0 & -\frac{I_{21}}{r I_{22}} & -\frac{\alpha^{2} K_{0}}{r I_{22}} & 0 \\
0 & \frac{4 \alpha K_{33}}{K_{0}} & 0 & \alpha & 0 & 0 & 0 & 0 \\
A_{41} & 0 & \frac{\alpha K_{12}}{K_{22}} & 0 & 0 & 0 & \frac{1}{r} & 0 \\
-\frac{1}{r} & 0 & 0 & 0 & 0 & \alpha \frac{I_{21}}{I_{22}} & \frac{\alpha^{3} K_{0}}{I_{22}} & 0 \\
0 & 2 \alpha^{2} \frac{K_{33}}{r I_{33}} & 0 & 0 & -\alpha & 0 & 0 & \frac{\alpha^{3} K_{0}}{I_{33}} \\
0 & 0 & 0 & -\frac{1}{r} & 0 & 0 & 0 & \alpha \\
0 & 0 & 0 & 0 & 0 & A_{86} & -\alpha \frac{I_{12}}{I_{22}} & 0
\end{array}\right)\left(\begin{array}{c}
{ }^{*} w_{S} \\
{ }^{*} \varphi_{S \varphi} \\
{ }^{*} M_{S \varphi} \\
{ }^{*} V_{S \varphi} \\
{ }^{*} v_{S} \\
{ }^{*} N_{S} \\
{ }^{*} N_{S \varphi x}
\end{array}\right)
$$

or

$$
\frac{\mathrm{d} Z_{S}}{r \mathrm{~d} \varphi}=Z_{S}^{\cdot}=A_{S} \cdot Z_{S}
$$

Here, $\alpha \equiv \frac{m \pi}{a}$

$$
\begin{gathered}
I_{11}=I_{22}=\frac{E t}{1-\mu^{2}}, \quad I_{21}=I_{12}=\frac{\mu E t}{1-\mu^{2}}, \quad I_{33}=\frac{E t}{2(1+\mu)} \\
K_{11}=K_{22}=\frac{E t^{3}}{24\left(1-\mu^{2}\right)}, \quad K_{21}=K_{12}=\frac{\mu E t^{3}}{24\left(1-\mu^{2}\right)}, \\
K_{33}=K_{34}=K_{43}=K_{44}=\frac{E t^{3}}{24(1+\mu)} \\
A_{41}=\frac{\alpha}{K_{0}}\left(K_{11}-\frac{K_{12} K_{21}}{K_{22}}-\frac{\pi^{2} k K_{0}}{\alpha^{2} b^{2}}\right), \quad A_{86}=-\frac{1}{\alpha K_{0}}\left(\frac{I_{12} I_{21}}{I_{22}}-I_{11}\right)
\end{gathered}
$$

Integrating Equation (2), the field transfer matrix $F_{S}$ is obtained as follows

$$
Z_{S}=\exp \left(A_{S} r \varphi\right) \cdot Z_{0}=F_{S} \cdot Z_{0}
$$

where

$$
\exp \left(A_{S} \varphi\right)=I+\left(A_{S} \varphi\right)+\frac{1}{2 !}\left(A_{S} \varphi\right)^{2}+\frac{1}{3 !}\left(A_{S} \varphi\right)^{3}+\cdots
$$


$I$ is the unit matrix.

\subsection{Field Transfer Matrix for Plate-Panel}

During the equilibrium equations of forces for the plate-panel subjected to in-plane compressive force and considering the relations between strains and deformations for plate-panel (Figure 2), the partial differential equations for the state variables ${ }^{*} w_{P},{ }^{*} \varphi_{P y},{ }^{*} M_{P y},{ }^{*} V_{P y},{ }^{*} v_{P},{ }^{*} u_{P},{ }^{*} N_{P y}$ and ${ }^{*} N_{P y x}$ are obtained. By substituting the extended the state variables on the basis of the condition at the simple support of the plate-panel into the partial differential equations, the following ordinary differential equations

$$
\frac{\mathrm{d} Z_{P}}{\mathrm{~d} y}=Z_{P}^{\cdot}=\left[{ }^{*}{w_{P}}^{\cdot},{ }^{*} \varphi_{P y}^{\cdot},{ }^{*} M_{P y}^{\cdot},{ }^{*} V_{P y}^{\cdot},{ }^{*} v_{P}^{\cdot},{ }^{*} u_{P},{ }^{*} N_{P y}^{\cdot},{ }^{*} N_{P y x}^{\cdot}\right]^{\mathrm{T}}
$$

Referred to the variable $y$ are obtained only:

$$
\left(\begin{array}{c}
{ }^{*} w_{P} \\
{ }^{*} \varphi_{P y} \\
{ }^{*} M_{P y} \\
{ }^{*} V_{P y} \\
{ }^{*} v_{P} \\
{ }^{*} u_{P} \\
{ }^{*} N_{P y} \\
{ }^{*} N_{P y x}
\end{array}\right)=\left(\begin{array}{cccccccc}
0 & -\alpha & 0 & 0 & \frac{1}{r} & 0 & 0 & 0 \\
-\frac{\alpha K_{21}}{K_{22}} & 0 & \frac{\alpha K_{0}}{K_{22}} & 0 & 0 & 0 & 0 & 0 \\
0 & \frac{4 \alpha K_{33}}{K_{0}} & 0 & \alpha & 0 & 0 & 0 & 0 \\
A_{41} & 0 & \frac{\alpha K_{12}}{K_{22}} & 0 & 0 & 0 & 0 & 0 \\
0 & 0 & 0 & 0 & 0 & \alpha \frac{I_{21}}{I_{22}} & \frac{\alpha^{3} K_{0}}{I_{22}} & 0 \\
0 & 0 & 0 & 0 & -\alpha & 0 & 0 & \frac{\alpha^{3} K_{0}}{I_{33}} \\
0 & 0 & 0 & 0 & 0 & 0 & 0 & \alpha \\
0 & 0 & 0 & 0 & 0 & A_{86} & -\alpha \frac{I_{12}}{I_{22}} & 0
\end{array}\right)\left(\begin{array}{c}
{ }^{*} w_{P} \\
{ }^{*} \varphi_{P y} \\
{ }^{*} M_{P y} \\
{ }^{*} V_{P y} \\
{ }^{*} v_{P} \\
{ }^{*} u_{P} \\
{ }^{*} N_{P y} \\
{ }_{P y x}
\end{array}\right)
$$

or

$$
\frac{\mathrm{d} Z_{P}}{\mathrm{~d} y}=Z_{P}^{\cdot}=A_{P} \cdot Z_{P}
$$
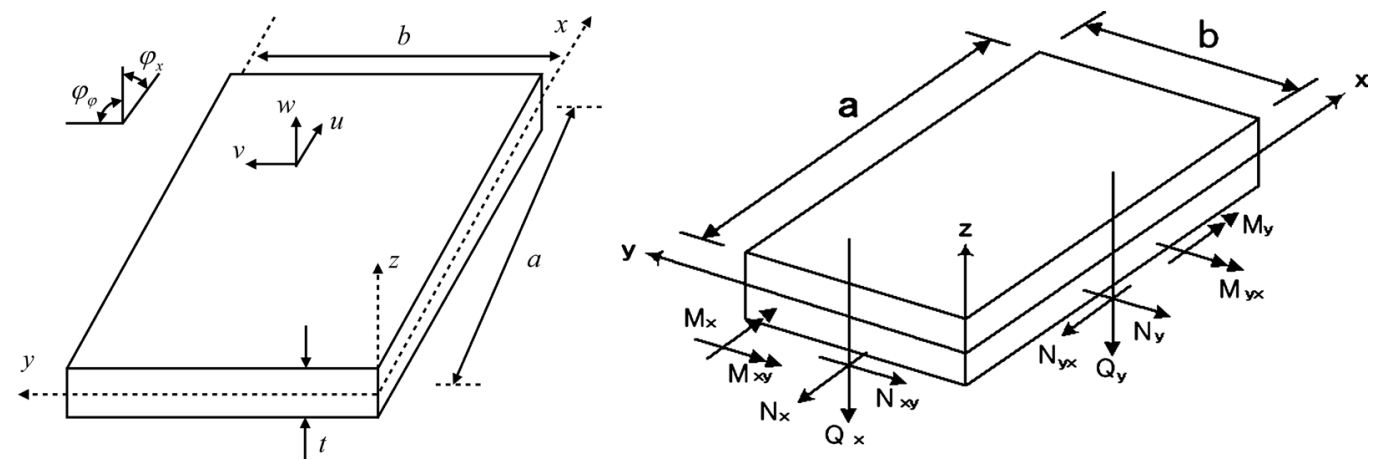

Figure 2. Forces and deformations of plate-panel subjected to in-plane compressive load. 
Integrating Equation (7), the field transfer matrix $F_{P}$ is obtained as follows:

$$
Z_{P}=\exp \left(A_{P} y\right) \cdot Z_{0}=F_{P} \cdot Z_{0}
$$

where

$$
\exp \left(A_{P} y\right)=I+\left(A_{P} y\right)+\frac{1}{2 !}\left(A_{P} y\right)^{2}+\frac{1}{3 !}\left(A_{P} y\right)^{3}+\cdots
$$

$I$ is the unit matrix.

Here, by considering $r \cong \infty$ and $y=r \varphi$, the partial differential equations of plate-panel Equation (7) is obtained from the partial differential equations Equation (2) of shell-panel.

\subsection{The Field Point Matrix for Thin-Walled Members}

As shown in Figure 3 the state vectors for each panel are referred to the local coordinate system. Therefore the relations between the state vectors of two consecutive panels are required, in order to process the transfer procedures of state vectors over these panels. Considering the relation between the state vectors of two plate panels (Figure 3), the following equations is obtained

$$
\left(\begin{array}{l}
{ }^{*} w \\
{ }^{*} \varphi_{y} \\
{ }^{*} M_{y} \\
{ }^{*} V_{y} \\
{ }^{*} v \\
{ }^{*} u \\
{ }^{*} N_{y} \\
{ }^{*} N_{y x}
\end{array}\right)^{L}=\left(\begin{array}{cccccccc}
\cos \theta & 0 & 0 & 0 & -\sin \theta & 0 & 0 & 0 \\
0 & 1 & 0 & 0 & 0 & 0 & 0 & 0 \\
0 & 0 & 1 & 0 & 0 & 0 & 0 & 0 \\
0 & 0 & 0 & \cos \theta & 0 & 0 & -\sin \theta & 0 \\
\sin \theta & 0 & 0 & 0 & \cos \theta & 0 & 0 & 0 \\
0 & 0 & 0 & 0 & 0 & 1 & 0 & 0 \\
0 & 0 & 0 & \sin \theta & 0 & 0 & \cos \theta & 0 \\
0 & 0 & 0 & 0 & 0 & 0 & 0 & 1
\end{array}\right)\left(\begin{array}{c}
{ }^{*} w \\
{ }^{*} \varphi_{y} \\
{ }^{*} M_{y} \\
{ }^{*} V_{y} \\
{ }^{*} v \\
{ }^{*} u \\
{ }^{*} N_{y} \\
{ }^{*} N_{y x}
\end{array}\right)^{R}
$$

or

$$
{ }^{*} Z_{i}^{L}=P_{i} \cdot{ }^{*} Z_{i}^{R}
$$

where, $P_{i}$ is the field point matrix relating the state vectors between two consecutive panels.

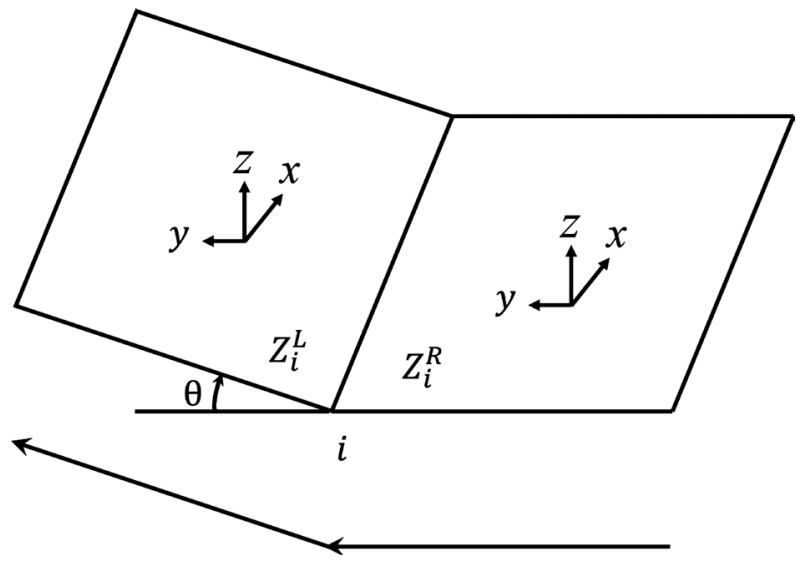

Figure 3. Relation between consecutive panels. 


\section{Stability Equation}

Performing the transfer procedure from section 0 to 10 on lipped channel column with shell-shaped grooves in the web and the flanges, the relation between the initial state vector, $Z_{0}$, and that at section $10, Z_{10}$ is described as follows (Figure 4(a)):

$$
\begin{aligned}
Z_{10}= & F_{P 10} \cdot P_{9} \cdot F_{P 9} \cdot P_{8} \cdot F_{S 8} \cdot P_{7} \cdot F_{P 7} \cdot P_{6} \cdot F_{P 6} \cdot P_{5} \cdot F_{S 5} \\
& \cdot P_{4} \cdot F_{P 4} \cdot P_{3} \cdot F_{P 3} \cdot P_{2} \cdot F_{S 2} \cdot P_{1} \cdot F_{P 1} \cdot P_{0} \cdot F_{P 0} \cdot Z_{0}
\end{aligned}
$$

where, $F_{p}$ and $F_{S}$ are the field transfer matrices, and $P$ is the point transfer matrix. Similarly, the transfer procedure is performed on the lipped channel column with triangle-shaped grooves in the web and the flanges (Figure 4(b)).

$$
\begin{aligned}
Z_{13}= & F_{P 13} \cdot P_{12} \cdot F_{P 12} \cdot P_{11} \cdot F_{P 11} \cdot P_{10} \cdot F_{P 10} \cdot P_{9} \cdot F_{P 9} \cdot P_{8} \cdot F_{P 8} \cdot P_{7} \cdot F_{P 7} \cdot P_{6} \cdot F_{P 6} \\
& \cdot P_{5} \cdot F_{P 5} \cdot P_{4} \cdot F_{P 4} \cdot P_{3} \cdot F_{P 3} \cdot P_{2} \cdot F_{P 2} \cdot P_{1} \cdot F_{P 1} \cdot P_{0} \cdot F_{P 0} \cdot Z_{0}
\end{aligned}
$$

\section{Numerical Analysis and Analytical Results}

For the purpose of grasping fundamental mechanical behavior on cross section of folded and curved grooves the buckling strength and the buckling mode shape of the thin-walled channel section columns with stiffeners subjected to axial compressive force are investigated by use of the transfer matrix method.

The transfer matrix method is analytical method to perform analysis multiplying the field transfer matrix derived by the governing equations for plate- or shell-panel composing the thin-walled member and the transform coordinates matrix relating the vectors between plate-panels composing the thin-walled members, and owing to finish construction of transfer matrices a time the structural analysis is consequential to multiplication of matrices only and is very automatically advanced to be not necessary intervention of mechanical principle during analysis [4]. Therefore, as compared with the other numerical analysis method of the finite element method and so the transfer matrix method is possible and efficient analysis method to obtain very accurate solution in small number

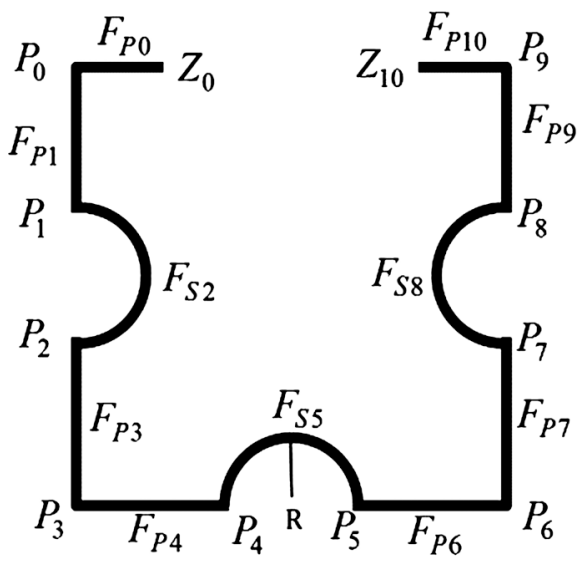

(a)

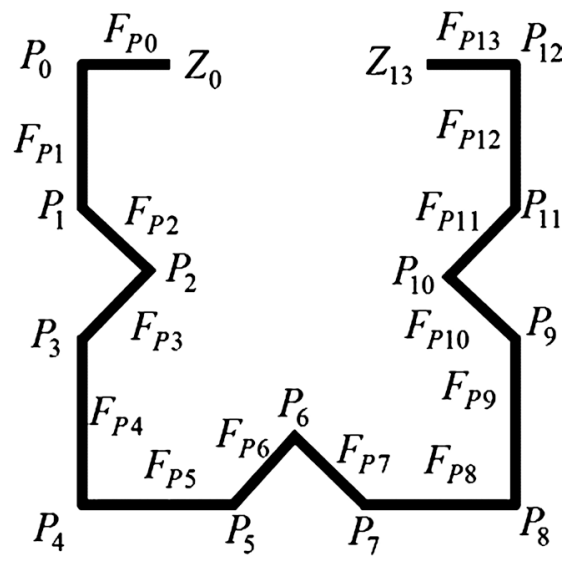

(b)

Figure 4. Lipped channel column with curved and folded grooves in the web and the flanges. (a) shell-shaped grooves; (b) triangle-shaped grooves. 
of variables notably.

Although the present method is naturally a solution procedure for one-dimensional problems, this method is extended to two-dimensional problems by introducing the trigonometric series into the governing equations of problems. Further this transfer matrix method is expanded for the thin-walled member by introducing the field transfer matrix and the transform coordinates matrix relating the vectors between plate-panels composing the thin-walled member. In present paper the buckling analysis is generated by introducing the field transfer matrix and the transform coordinates matrix on lipped channel section columns with folded and curved grooves.

In Figure 5 analytical models are shown. Model A are the lipped channel section columns without stiffener. Model B are the lipped channel section columns with triangle-shaped folded grooves or shell-shaped curved grooves to web. Model C are the lipped channel section columns with triangle-shaped folded grooves or shell-shaped curved grooves to flange, and Model D are the lipped channel section with triangle-shaped folded grooves or shell-shaped curved grooves to both web and flange. In Table 1 the cross sections and the size of analytical models are shown.

In Figure 6 the buckling coefficients of normal lipped channel section columns without stiffener are shown during various length of lip (L) in member aspect ratio $(\mathrm{a} / \mathrm{b})$, and the local buckling behavior are different from lip length. In the case of $\mathrm{L}=15 \mathrm{~mm}$ and $20 \mathrm{~mm}$ behavior of local and overall buckling are generated. In the case of $\mathrm{L}=10 \mathrm{~mm}$ the local buckling behavior is shown in

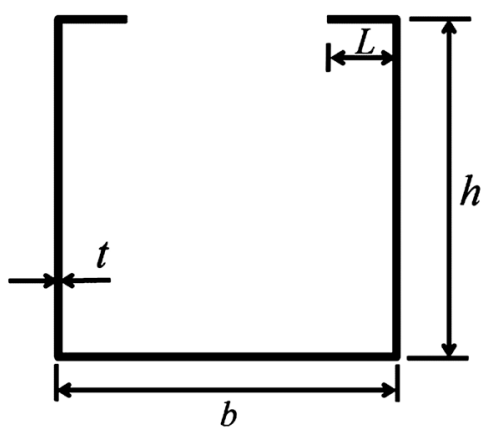

Type A

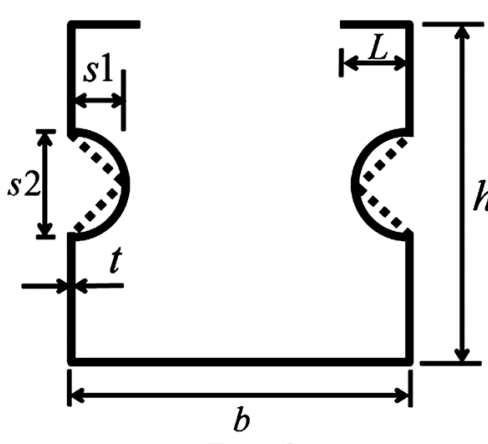

Type C

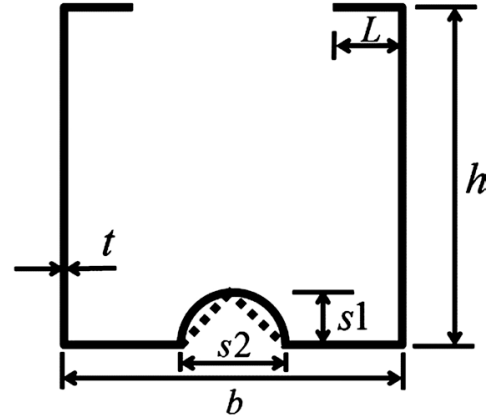

Type B

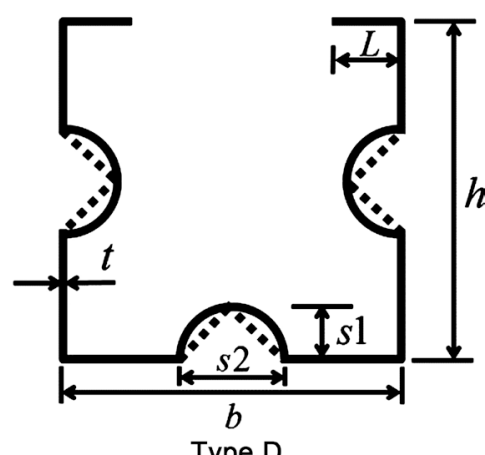

Type D

Figure 5. Analytical models. 
Table 1. Parameters of analytical models.

\begin{tabular}{ccccccc}
\hline specimens & $\mathrm{t}(\mathrm{mm})$ & $\mathrm{b}(\mathrm{mm})$ & $\mathrm{h}(\mathrm{mm})$ & $\mathrm{S} 1(\mathrm{~mm})$ & $\mathrm{S} 2(\mathrm{~mm})$ & $\mathrm{L}(\mathrm{mm})$ \\
\hline $\mathrm{A}$ & 1.3 & 130 & 130 & - & - & $5,10,15,20$ \\
B-shell & 1.3 & 130 & 130 & 10 & 20 & $5,10,15,20$ \\
B-triangle & 1.3 & 130 & 130 & 10 & 20 & $5,10,15,20$ \\
C-shell & 1.3 & 130 & 130 & 10 & 20 & $5,10,15,20$ \\
C-triangle & 1.3 & 130 & 130 & 10 & 20 & $5,10,15,20$ \\
D-shell & 1.3 & 130 & 130 & 10 & 20 & $5,10,15,20$ \\
D-triangle & 1.3 & 130 & 130 & $5,10,15,20$ & $10,20,30,40$ & $5,10,15,20$ \\
E-shell & 0.42 & 120 & 90 & 10 & 20 & 12 \\
E-triangle & 0.42 & 120 & 90 & 10 & 20 & 12 \\
\hline
\end{tabular}

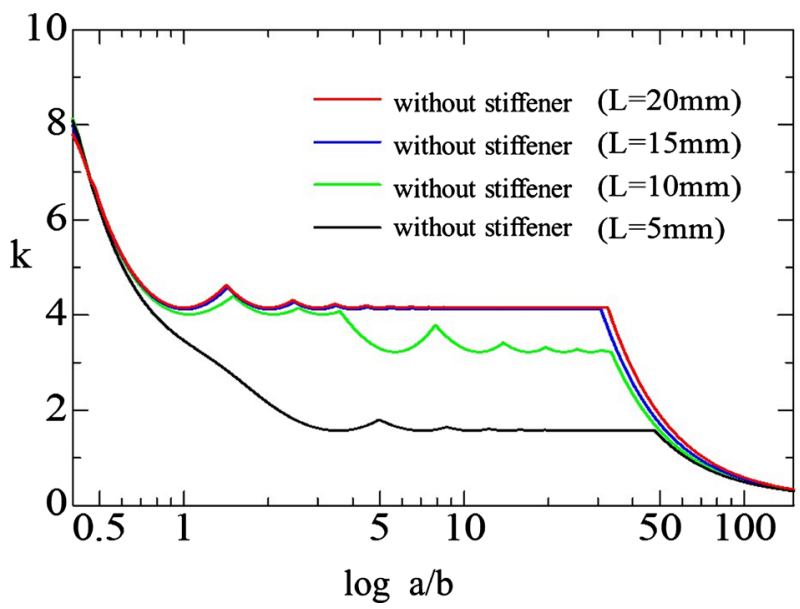

Figure 6. Buckling coefficients of lipped channel section columns without stiffener.

small member aspect ratio $(\mathrm{a} / \mathrm{b})$. However, before sifting over to overall buckling the distortion buckling is produced, and after that the buckling behavior is sifting over to overall buckling. In the case of $\mathrm{L}=5 \mathrm{~mm}$ local and overall buckling behavior is produced failing of buckling strength in comparison with that of lip length $\mathrm{L}=15 \mathrm{~mm}$ and $20 \mathrm{~mm}$.

In Figure 7 the buckling coefficients of lipped channel section columns with various shell-shaped grooves are shown in member aspect ratio $(a / b)$ under length of lip ( $\mathrm{L}=20 \mathrm{~mm}$ ). In the case of $\mathrm{S} 1=10 \mathrm{~mm}, 15 \mathrm{~mm}$ and $20 \mathrm{~mm}$ behavior of local buckling are generated in small member aspect ratio $(\mathrm{a} / \mathrm{b})$. Before sifting over to overall buckling the distortional buckling are produced, and after that the buckling behavior is sifting over to overall buckling. In the case of $S 1=5$ $\mathrm{mm}$ the fall of buckling strength by local and overall buckling behavior are produced in comparison with that of groove-height $S 1=10 \mathrm{~mm}, 15 \mathrm{~mm}$ and $20 \mathrm{~mm}$. The buckling strength of groove-height $\mathrm{S} 1=10 \mathrm{~mm}$ is a little failing in comparison with those of $\mathrm{S} 1=15 \mathrm{~mm}$ and $20 \mathrm{~mm}$. Then, as groove-web ratio $(2 \mathrm{~S} 1 / \mathrm{b}=$ $\mathrm{S} 2 / \mathrm{b}$ ) is about $0.15-0.20$ by Hancock [1], the groove height (S1) is used with 10 
$\mathrm{mm}$ in this study from now on.

In Figure 8 the buckling coefficients of lipped channel section columns with shell-shaped curved groove in the web are shown during various length of lip and member aspect ratio. Owing to stiffening the web the buckling coefficients are increasing under $\mathrm{L}=15 \mathrm{~mm}$ and $20 \mathrm{~mm}$. However, under $\mathrm{L}=5 \mathrm{~mm}$ and 10 $\mathrm{mm}$ the buckling strength are not increasing, and as this reason the local buckling in the flanges are produced. Therefore, it is considered that stiffened effect is not generated by shell shaped groove in the web.

In Figure 9 and Figure 10 the buckling coefficients of lipped channel section with triangle-shaped folded grooves and shell-shaped curved groove in the flanges are shown during member aspect ratio and various length of lip respectively. As compared with both figures the local buckling strength is not almost difference in local buckling range. However, in distortional buckling range the strength of member with shell-shaped curved grooves is increasing in comparison

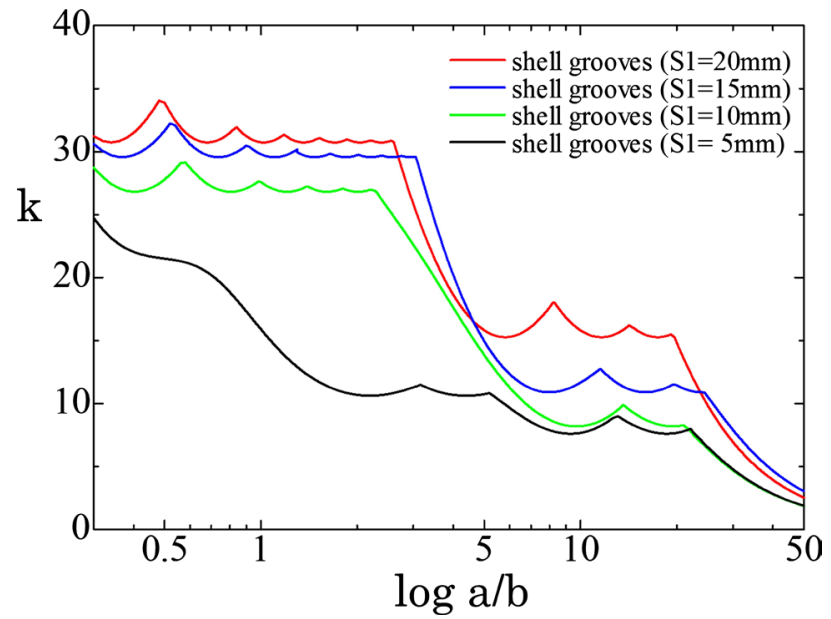

Figure 7. Buckling coefficients of lipped channel section columns with various shell-shaped grooves under lipped length $\mathrm{L}=20 \mathrm{~mm}$.

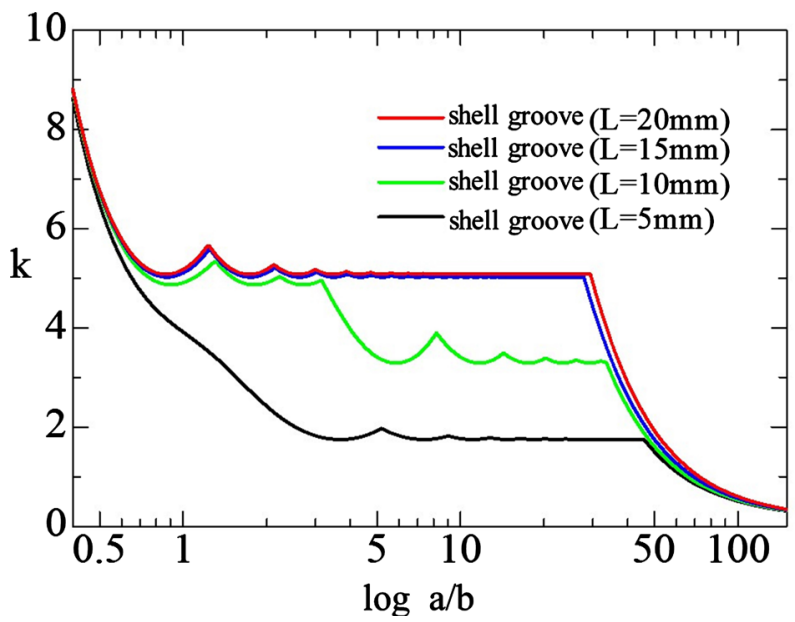

Figure 8. Buckling coefficients of lipped channel section columns with shell-shaped groove in the web. 


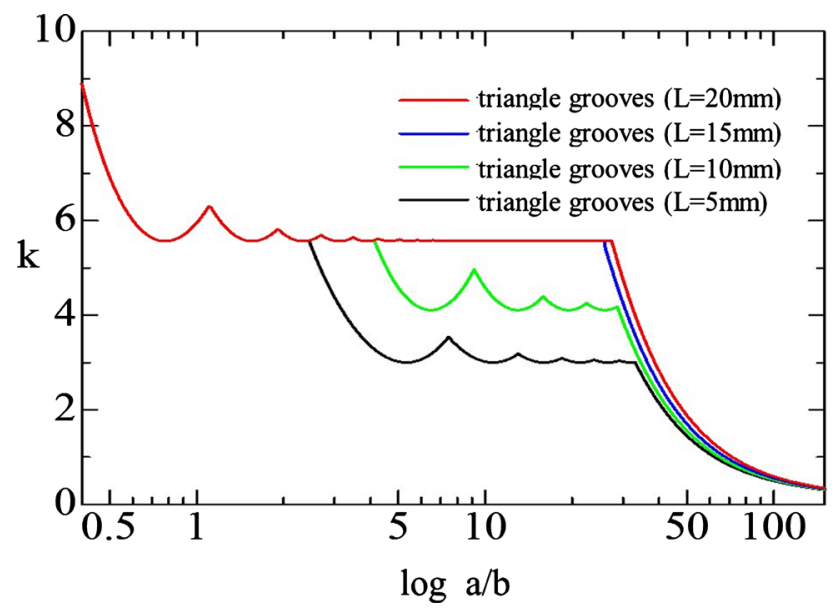

Figure 9. Buckling coefficients of lipped channel section columns with triangle-shaped grooves in the flanges.

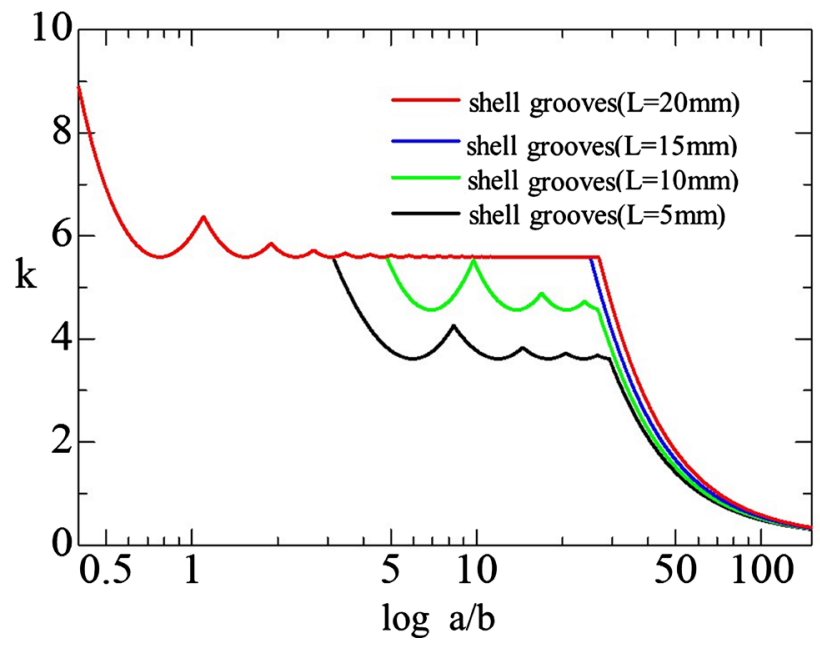

Figure 10. Buckling coefficients of lipped channel section columns with shell-shaped grooves in the flanges.

with that with triangle-shaped folded grooves. As compared with the local and distortional buckling strength of Figure 8 stiffening the web by shell shaped groove, that of Figure 10 stiffening the flanges by shell shaped grooves is naturally appreciated to be increasing.

In Figure 11 the buckling mode shape of lipped channel section column with shell-shaped curved grooves in the flanges for $\mathrm{a} / \mathrm{b}=2, \mathrm{~m}=2$ exhibit the local buckling mode, and symmetric local buckling mode of the cross section is recognized. By the existing state the case producing anti-symmetric local buckling mode is also in existence. As comparison with the buckling strength, that of symmetric buckling is enlarging.

In Figure 12 the buckling mode shape of lipped channel section column with shell-shaped curved grooves in the flanges for $\mathrm{a} / \mathrm{b}=10, \mathrm{~m}=2$ exhibit the distortional buckling mode, and it is appreciated that the flanges are deforming to the inside or the outside by means of member aspect ratio. 


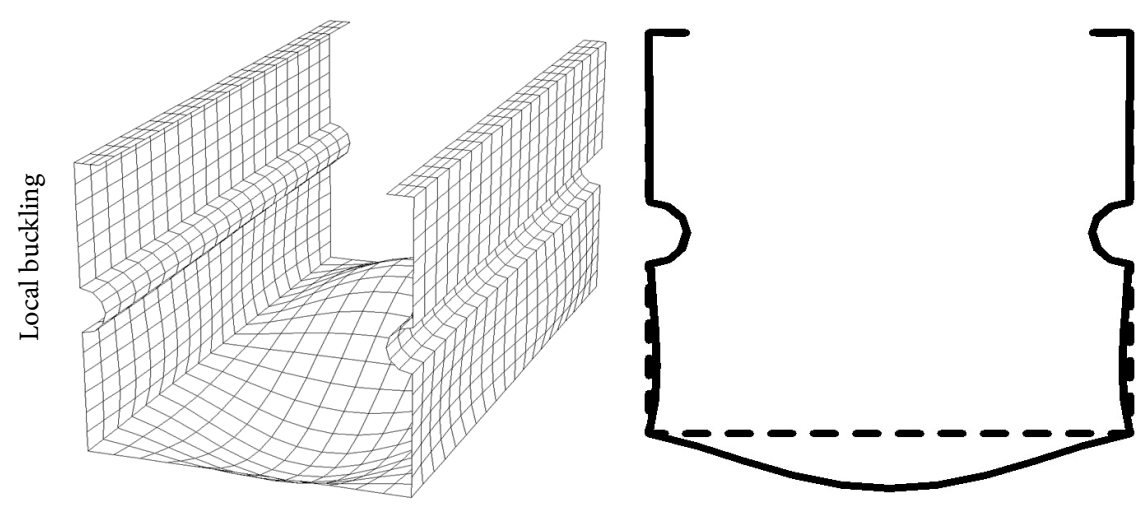

$\mathrm{a} / \mathrm{b}=2, \mathrm{~m}=2$

Figure 11. Local buckling mode shape of lipped channel section column with shell-shaped curved grooves in the flanges.

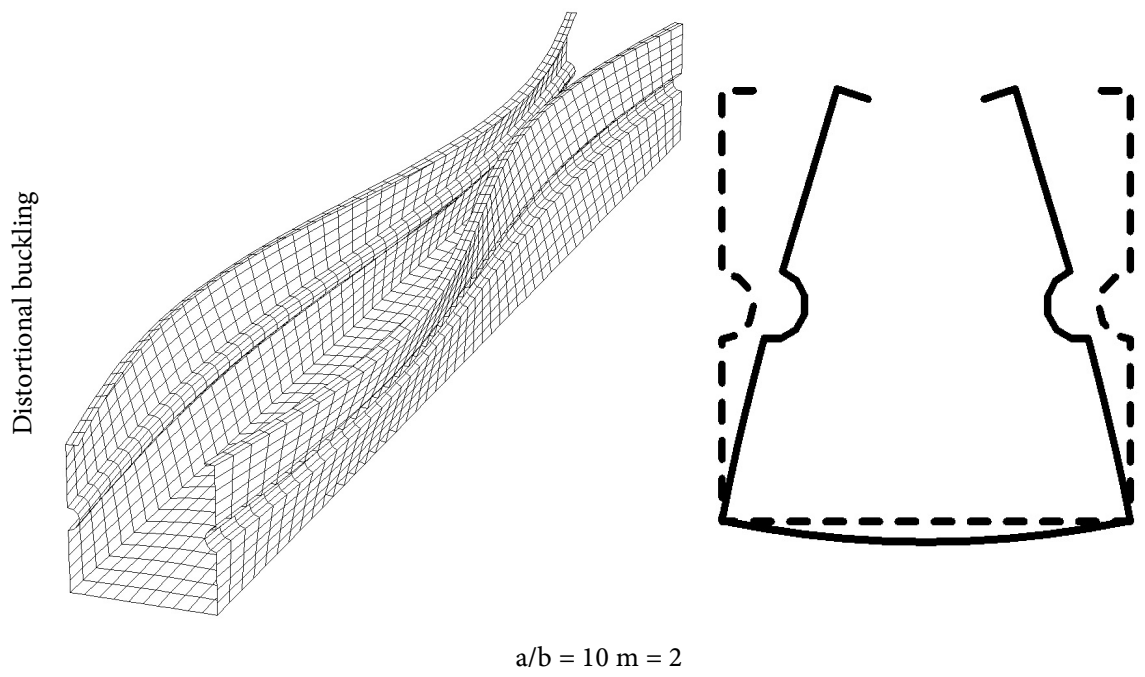

Figure 12. Distortional buckling mode shape of lipped channel section column with shell-shaped curved grooves in the flanges.

Figure 13 shows the buckling coefficients of lipped channel section with shell-shaped curved grooves in the web and the flanges during various length of lip (L) and member aspect ratio (a/b). As shown in Figure 13, the local buckling strength is naturally rising with shell-shaped curved grooves in local range. However, as falling rapidly after that the distortional buckling is produced, and then the effect of shell-shaped grooves are shown on the local buckling

In Figure 14 the buckling coefficients of lipped channel section with triangle-shaped folded grooves in the web and the flanges are shown for various length of lip (L) and member aspect ratio (a/b). As shown in Figure 14, the same tendency is shown in respect of member with shell-shaped curved groove. As regards the buckling strength, that of the member with shell-shaped curved grooves is rising in comparison with the member with triangle shaped folded grooves. 


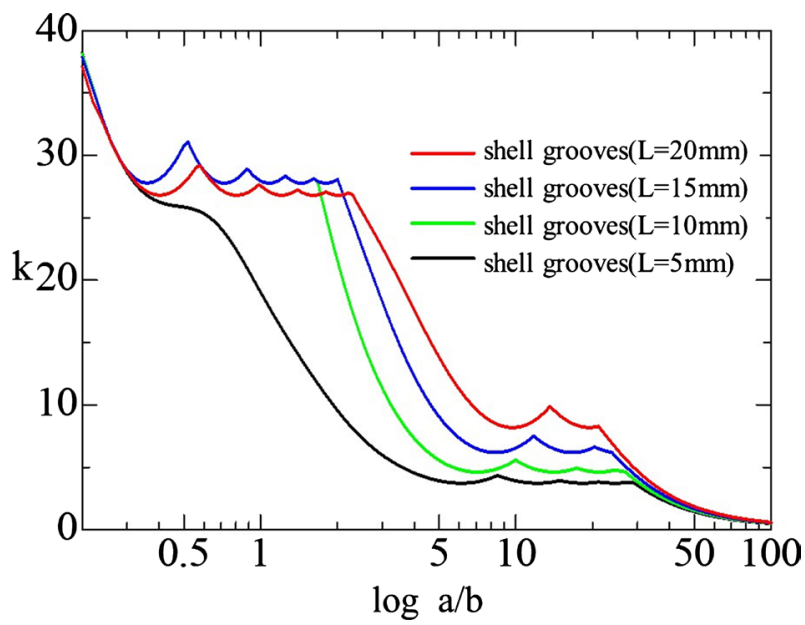

Figure 13. Buckling coefficients of lipped channel section columns with shell-shaped curved grooves in the flanges and web.

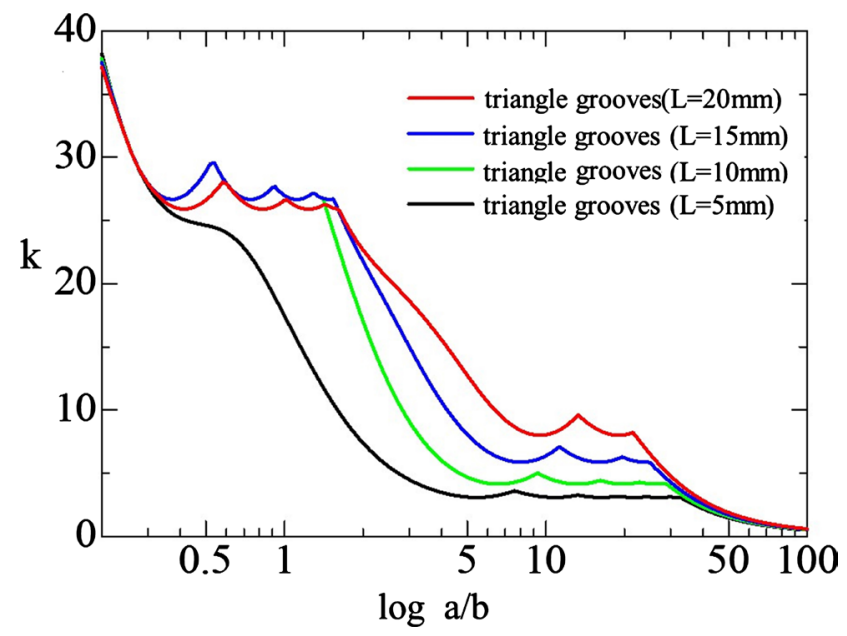

Figure 14. Buckling coefficients of lipped channel section columns with triangle-shaped folded grooves in the flanges and web.

In Figure 15 the buckling mode shape of lipped channel section column with shell-shaped curved grooves in the flanges and the web for $\mathrm{a} / \mathrm{b}=1, \mathrm{~m}=3$ and $\mathrm{L}$ $=10 \mathrm{~mm}$ exhibits the local buckling mode, and symmetric local buckling mode of the cross section is recognized.

In Figure 16 the distortional buckling mode shape of lipped channel section column with shell-shaped curved grooves in the flanges and the web is shown under $\mathrm{a} / \mathrm{b}=10, \mathrm{~m}=2$ and $\mathrm{L}=10 \mathrm{~mm}$, and it is appreciated that the flanges are deforming to the inside or the outside by means of member aspect ratio.

This analytical model with triangle-shaped folded grooves is same the model (Model E) calculated by Hancock. In Figure 17 the buckling coefficients of lipped channel section columns with triangle- or shell-shaped grooves in the web and the flanges subjected to uniform axial load are shown under member aspect ratios $(\mathrm{a} / \mathrm{b}=1.0-120.0)$. Further, the buckling coefficient of lipped channel section columns with triangle-shaped grooves in the web and the flanges obtained 


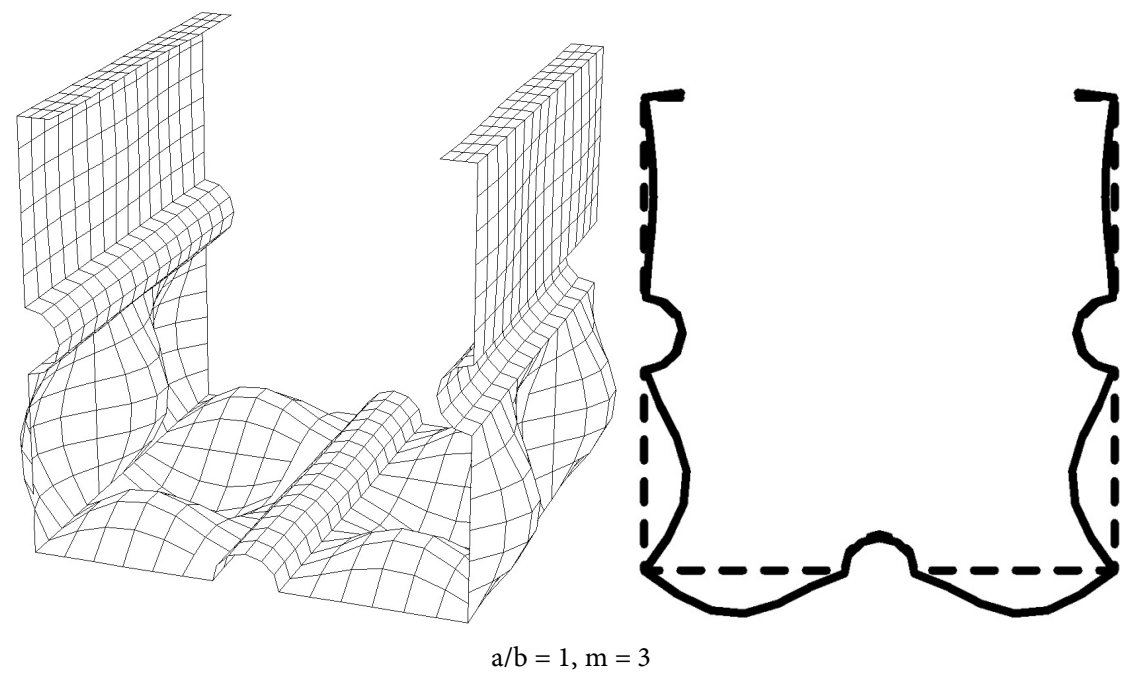

Figure 15. Local buckling mode shape of lipped channel section column with shell-shaped curved grooves in the flanges and web.

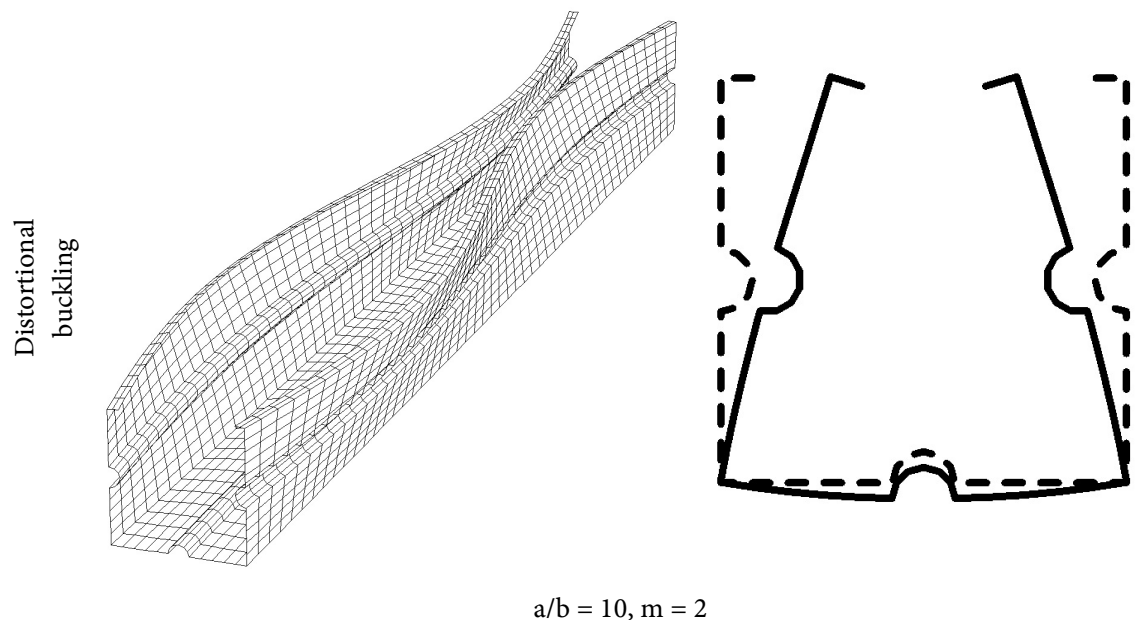

Figure 16. Distortional buckling mode shape of lipped channel section column with shell-shaped curved grooves in the flanges and web.

by Hancock and Yang during finite strip method are simultaneously shown in local and distortional regions, and then the buckling coefficients of normal lipped channel section column without stiffener is together shown as comparison. As shown in Figure 17, one of three types of local, distortional, and overall buckling behavior is generated in respective length of lipped channel section column with triangle- or shell-shaped grooves in the web and the flanges. And so the wave-length of distortional buckling is intermediate between that of local buckling and overall buckling. In comparison with the buckling strength obtained by Hancock and Yang [1] and that obtained by the transfer matrix method, the buckling strength is almost equal except some differences in distortional region. In distortional region the effect of stiffener on lipped channel column with shell-shaped curved grooves in the web and the flanges is large in 


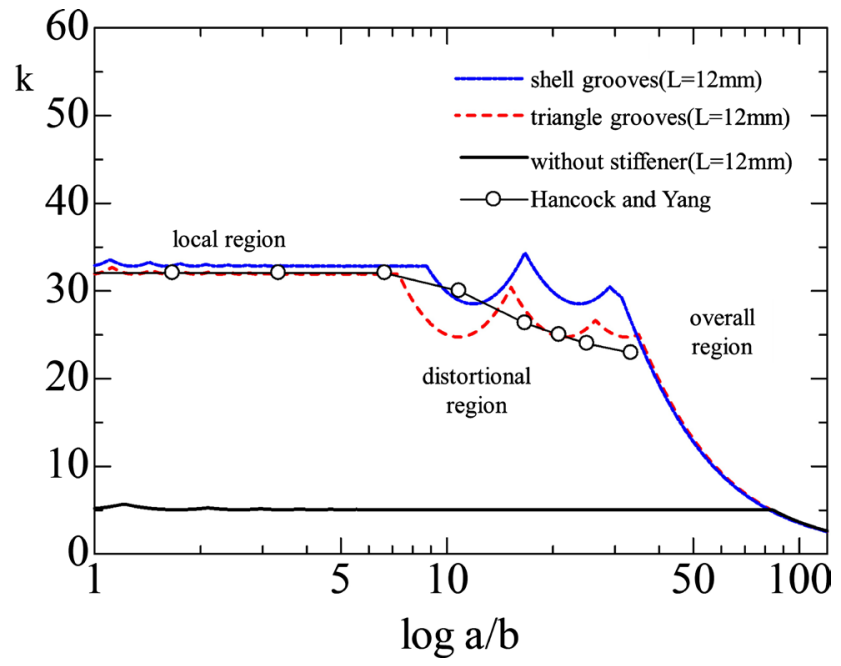

Figure 17. Comparison of the buckling strength of lipped channel section columns with triangle- and shell-shaped grooves obtained by transfer matrix method and finite strip method.

comparison with that on lipped channel column with triangle-shaped folded grooves in the web and the flanges.

\section{Conclusions}

In this chapter a new cross section of shell-shaped curved groove was proposed instead of the thin-walled lipped channel section column with triangle-and rectangle-shaped folded grooves used ordinarily, and therefore the comparison and the examination of buckling strength and buckling behavior were generated in the case of preparing triangle-shaped folded and shell-shaped curved grooves to the web and the flanges of thin-walled channel section column. As compared with the buckling strength stiffening the web, that stiffening the flanges is naturally appreciated to be increasing.

Regarding to the buckling strength, that of the member with shell-shaped curved groove is a little rising in comparison with the member with triangle shaped folded groove.

Therefore, it is considered that the use of the shell-shaped curved groove is possible sufficiently.

Then, it is considered that there are sufficient possibilities when using this thin-walled lipped channel section with shell-shaped curved grooves as compression.

\section{Conflicts of Interest}

The authors declare no conflicts of interest regarding the publication of this paper.

\section{References}

[1] Kwon, Y.B., Kim, B.S. and Hancock, G.J. (2009) Compression Tests of High Strength Cold-Formed Steel Channels with Buckling Interaction. Journal of Con- 
structional Steel Research, 65, 278-289. https://doi.org/10.1016/j.jcsr.2008.07.005

[2] Wang, C.G. and Bai, Y. (2016) Method Considered Distortional-Local Interaction Buckling ofr Bearing Capacity of Channels with Complex Edge Stiffeners and Web stiffeners under Axial Compression. MATEC Web of Conferences, Vol. 67.

[3] Hoshide, K., Ohga, M. and Shigematsu, T. (2017) Study on Elastic Buckling Behavior in Steel Cylindrical Shells with Folded and Curved Grooves. Journal of Civil Engineering and Architecture Research, 4, 1951-1961.

[4] Ohga, M., Kwaguchi, K. and Shigematu, T. (1995) Buckling Analysis of Thin Walled Members with Closed Cross Sections. Thin-Walled Structures, 22, 51-70. https://doi.org/10.1016/0263-8231(95)95937-V

[5] Shigematsu, T., Ohga, M. and Hara, T. (1992) Ermittlung der Beultraglasten ausgesteifter Schalen mit Hilfe von Übertragungsmatrizen. Archive of Applied Mechanics, 62, 28-42. https://doi.org/10.1007/BF00786679 